\title{
Electronic, vibrational, elastic, and piezoelectric properties of monolayer Janus MoSTe phases: A first-principles study
}

\author{
M. Yagmurcukardes, ${ }^{1, *}$ C. Sevik, ${ }^{2}$ and F. M. Peeters ${ }^{1}$ \\ ${ }^{1}$ Department of Physics, University of Antwerp, Groenenborgerlaan 171, B-2020 Antwerp, Belgium \\ ${ }^{2}$ Department of Mechanical Engineering, Faculty of Engineering, Eskişehir Technical University, Eskisehir, TR 26555, Turkey
}

(Received 13 May 2019; published 19 July 2019)

\begin{abstract}
By performing density functional theory based first-principles calculations, the electronic, vibrational, elastic, and piezoelectric properties of two dynamically stable crystal phases of monolayer Janus MoSTe, namely $1 H$-MoSTe and $1 T^{\prime}$-MoSTe, are investigated. Vibrational frequency analysis reveals that the other possible crystal structure, $1 T$-MoSTe, of this Janus monolayer does not exhibit dynamical stability. The $1 H$-MoSTe phase is found to be an indirect band-gap semiconductor while $1 T^{\prime}$-MoSTe is predicted as small-gap semiconductor. Notably, in contrast to the direct band-gap nature of monolayers $1 H-\mathrm{MoS}_{2}$ and $1 H-\mathrm{MoTe}_{2}, 1 H-\mathrm{MoSTe}$ is found to be an indirect gap semiconductor driven by the induced surface strains on each side of the structure. The calculated Raman spectrum of each structure shows unique character enabling us to clearly distinguish the stable crystal phases via Raman measurements. The systematic piezoelectric stress and strain coefficient analysis reveals that out-of-plane piezoelectricity appears in $1 H$-MoSTe and the noncentral symmetric $1 T^{\prime}$-MoSTe has large piezoelectric coefficients. Static total-energy calculations show clearly that the formation of $1 T^{\prime}-\mathrm{MoSTe}$ is feasible by using $1 T^{\prime}-\mathrm{MoTe}_{2}$ as a basis monolayer. Therefore, we propose that the Janus MoSTe structure can be fabricated in two dynamically stable phases which possess unique electronic, dynamical, and piezoelectric properties.
\end{abstract}

DOI: 10.1103/PhysRevB.100.045415

\section{INTRODUCTION}

Following the successful exfoliation of graphene [1], interest in ultrathin two dimensional (2D) materials has been grown significantly. Among 2D monolayer materials, particular attention has been paid to the transition metal dichalcogenides (TMDs) family which hold potential for various applications such as semiconductor and optoelectronic devices due to their electronic properties ranging from metallic to semiconducting and even to superconducting [2-6].

For instance, layered $\mathrm{MoS}_{2}$, a famous member of the TMDs family, has been studied extensively owing to its distinctive electronic, optical, and mechanical properties [7-15]. In particular, due to its direct band gap, monolayer $\mathrm{MoS}_{2}$ has been thoroughly investigated as a potential candidate to be used in optoelectronic and photonic device applications. Monolayer $\mathrm{MoTe}_{2}$, another important member of TMDs, due to its tunable crystal phases, $1 H$ and $1 T^{\prime}$, possessing unique semiconducting and semimetallic properties [16,19], respectively, has been also deeply investigated. Recently, the theoretically estimated structural phase transition in $\mathrm{MoTe}_{2}$ between $1 H$ and $1 T^{\prime}$ has been reported experimentally through different techniques such as thermal synthesis at high temperatures [19], irreversible growth by element substitution [17], and laser irradiation [18]. Furthermore, such a structural phase transition has been achieved by electrostatic gating of $1 \mathrm{H}-\mathrm{MoTe}_{2}$ as a reversible process [20].

*mehmet.yagmurcukardes@uantwerpen.be
In addition to extensive research efforts on pure TMD crystals, recent advances in experimental techniques have allowed researchers to combine properties of different TMD monolayers in a single polar material, namely the Janus structure $[21,22]$. For instance, the top layer of monolayer $\mathrm{MoSe}_{2}$ has been experimentally replaced by $\mathrm{S}$ atoms and a Janus monolayer, namely MoSSe, has been fabricated. The realization of this compound monolayer has inspired many researchers to find a way to tune the physical properties of monolayer TMD crystals [21,22]. Afterward, energetic feasibility of other possible Janus monolayer crystals of different TMDs has been shown by first-principles studies [23-25]. Cheng et al. reported that intrinsic out-of-plane built-in electric field in monolayer Janus WSeTe leads to a significant Rashba spin splitting in its electronic band dispersion [24]. In addition, Dong et al. reported that the piezoelectric performance of Janus-type monolayer crystals is higher than commonly used monolayer materials [25].

Up to now, $1 H$ Janus TMD crystals, such as $\mathrm{MoS}_{2}$ and $\mathrm{MoTe}_{2}$, have been extensively investigated by first-principles calculations [25-30]. This crystal phase has been particularly taken into consideration in the studies due to the fact that it is a common crystalline form of monolayer TMDs and being the structure realized in the experiment. However, the construction of Janus crystals from two monolayer TMDs exhibiting different crystal symmetries such as $1 H$ and $1 T^{\prime}$ could pave the way to gain more control on novel materials synthesis. For instance, Sun et al. has very recently investigated the $1 T$-distorted phase of the Janus monolayer WSeTe and has reported similar unique physical properties as Janus MoSTe [31]. Therefore, in this study, inspiring from the already 
known ground-state octahedrally distorted $1 T$ structure of $\mathrm{MoTe}_{2}$, we systematically investigated possible Janus crystal structures of two well-known TMDs, $\mathrm{MoS}_{2}$ and $\mathrm{MoTe}_{2}$. We predict two dynamically stable MoSTe crystals possessing distinctive electronic, vibrational, and piezoelectric properties.

\section{COMPUTATIONAL METHODOLOGY}

For the first-principles calculations, the plane-wave basis projector augmented wave (PAW) method was employed in the framework of density-functional theory (DFT). The generalized gradient approximation (GGA) in the Perdew-BurkeErnzerhof (PBE) form [32,33] was used for the exchangecorrelation potential as implemented in the Vienna ab-initio Simulation Package (VASP) [34,35]. For the electronic-band structure calculations spin-orbit-coupling (SOC) was included with GGA functional and Heyd-Scuseria-Ernzerhof (HSE06) screened-nonlocal-exchange functional of the generalized Kohn-Sham scheme [36]. The van der Waals correction to the GGA functional was included by using the DFT-D2 method of Grimme [37]. Analysis of the charge transfers in the structures was determined by the Bader technique [38].

Electronic and geometric relaxations of the monolayers of $1 H$-MoSTe and $1 T$-distorted $\left(1 T^{\prime}\right)$ MoSTe were performed on three- and six-atom primitive unit cells, respectively. The kinetic-energy cutoff for plane-wave expansion was set to $500 \mathrm{eV}$ and the energy was minimized until its variation in the following steps became $10^{-8} \mathrm{eV}$. The Gaussian smearing method was employed for the total-energy calculations and the width of the smearing was chosen as $0.05 \mathrm{eV}$. Total Hellmann-Feynman forces was reduced down to $10^{-7} \mathrm{eV} / \AA$ for the structural optimization. $24 \times 24 \times 1$ and $15 \times 11 \times$ $1 \Gamma$ centered $k$-point grids were used for the both primitive unit cells, respectively. To avoid interaction between the neighboring layers, a vacuum space of $25 \AA$ was implemented in the calculations.

Phonon-dispersion curves were calculated by using the small displacement method as implemented in the PHON code [39]. The phonon frequencies and the corresponding offresonant Raman activities were calculated at the $\Gamma$ point of the Brillouin zone (BZ) using the small displacement method. Each atom in the primitive unit cell was initially distorted by $0.01 \AA$ and the corresponding dynamical matrix was constructed. Then, the vibrational modes were determined by a direct diagonalization of the dynamical matrix. The corresponding Raman activity of each phonon mode was obtained from the derivative of the macroscopic dielectric tensor by using the finite-difference method [40,41]. The relaxed-and clamped-ion elastic stiffness tensors were calculated by using the small displacement methodology as implemented in VASP. In addition, the piezoelectric stress coefficients were obtained directly using density functional perturbation theory (DFPT) with a sufficiently large $k$-point sampling and kinetic-energy cutoff values.

\section{1H AND 1T' PHASES OF MONOLAYER JANUS MOSTe}

The structural ground states of monolayers $\mathrm{MoS}_{2}$ and $\mathrm{MoTe}_{2}$ are known to be $1 H$ and $1 T^{\prime}$ phases, respectively while monolayers $\mathrm{MoS}_{2}$ and $\mathrm{MoTe}_{2}$ exhibit also dynamical stability in $1 T^{\prime}$ and $1 H$ phases. Therefore, the construction of both Janus $1 H$ - and $1 T^{\prime}$-MoSTe crystals should be feasible under appropriate experimental conditions. In the view of DFT-based ab-initio calculations, i.e., at $0 \mathrm{~K}$, it has been reported that the replacement of a heavier chalcogen atom with that of a lighter one is energetically more favorable [21,23]. In this context, the complete replacement of one surface of monolayer $1 T^{\prime}-\mathrm{MoTe}_{2}$ by $\mathrm{S}$ atoms seems to be more favorable and thus, the constructed monolayer Janus MoSTe is expected to crystallize in the octahedrally distorted $1 T$ phase (namely $\left.1 T^{\prime}\right)$. However, due to the dynamical stability of both $1 H$ and $1 T^{\prime}$ phases of the Janus MoSTe crystal, we present and discuss our results for both phases.

\section{A. Structural and vibrational properties}

For the construction of monolayer Janus MoSTe, three common structural phases, namely $1 H, 1 T$, and $1 T^{\prime}$, are considered and their dynamical stability is investigated via phonon frequency analysis through the whole BZ. As shown in Fig. 1(c), the calculated negative phonon frequencies corresponding to the low-lying acoustic mode clearly show the dynamical instability of the $1 T$ phase of monolayer Janus MoSTe. Indeed, it is somehow expected due to the dynamical instability of base monolayers, $\mathrm{MoS}_{2}$ and $\mathrm{MoTe}_{2}$ in this crystal form.

The other two structures, $1 H$ and $1 T^{\prime}$ are predicted as dynamically stable as presented in Figs. 1(b) and 1(d). Note
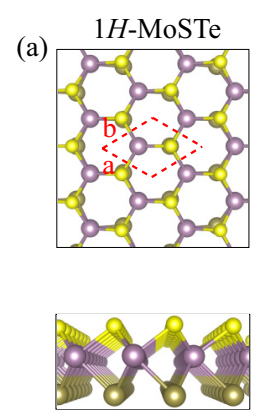
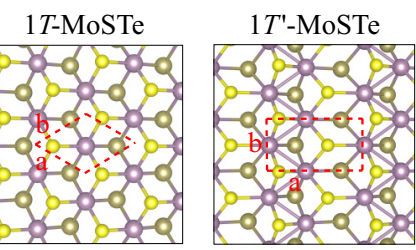

Mo $\bigcirc \mathrm{Te} \odot \mathrm{S}$
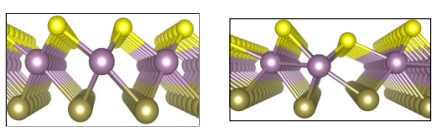
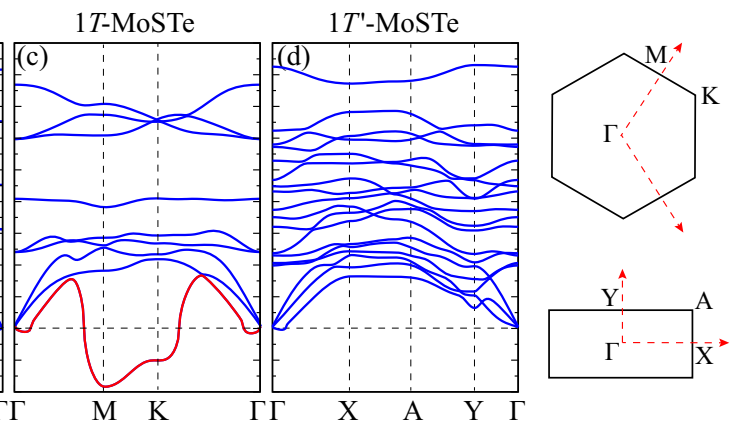

FIG. 1. For the three structural phases of monolayer Janus MoSTe crystal, (a) top and side views of the structures and (b)-(d) corresponding phonon band dispersions. The Brillouin zones of the hexagonal and rectangular lattices are given on the right-hand panel of the phonon bands. 
that the small negative frequencies in the out-of-plane acoustical (ZA) mode near the $\Gamma$ point are attributed to numerical artifacts caused by the small inaccuracies of the FFT grid. In $1 H$-MoSTe there are nine phonon branches, three of which are acoustic and in the $1 T^{\prime}$-MoSTe there are total of 18 phonon branches.

The optimized in-plane lattice parameters are calculated to be $a=b=3.36 \AA$ which are in between those for monolayer $1 H-\mathrm{MoS}_{2}(3.18 \AA)$ and monolayer $1 H-\mathrm{MoTe}_{2}(3.53 \AA)$. This structure is composed of a Mo layer sandwiched between $\mathrm{S}$ and Te layers separated antisymmetrically with respect to the Mo layer due to considerably different Mo-S and Mo-Te bond lengths, 2.43 and $2.72 \AA$, respectively. Therefore, two surfaces of this crystal possess different types of induced biaxial strains, i.e., the $\mathrm{S}$ side undergoes tensile strain whereas the Te side is compressed.

In the distorted crystal structure, $1 T^{\prime}$-MoSTe consists of covalently bonded S-Mo-Te layers in which Mo atoms form slightly buckled zigzag Mo-Mo chains running along the $b$ axis revealing the space group $C_{2 v}^{7}\left(P m n 2_{1}\right)$. The optimized anisotropic lattice parameters are predicted as $a=6.04$ and $b=3.32 \AA$. Due to the existence of Mo-Mo chains, different bond distances are calculated for each bond coordination (Mo-S and Mo-Te bond lengths vary between 2.392.58 and $2.69-2.82 \AA$, respectively). Thus, the same induced tensile and compressive biaxial strain exist in this structure as well.

Subsequent to the vibrational and structural analysis, the phonon frequencies and corresponding eigenfunctions are obtained at the $\Gamma$ point in order to calculate the first-order off resonant Raman spectrum via the change in dielectric tensor via individual mode displacement. The Raman spectrum calculations reveal that all of the six optical-phonon modes are Raman active in $1 H$-MoSTe due to the broken out-of-plane symmetry in this Janus crystal, as depicted in Fig. 2(a). Here, the calculated Raman spectra of $1 H$ phases of $\mathrm{MoS}_{2}$ and $\mathrm{MoTe}_{2}$ are also presented for comparison purposes. The two out-of-plane phonon modes having frequencies 408.4 and $226.5 \mathrm{~cm}^{-1}$ arise from the Mo-S $\left(A_{1}^{\mathrm{Mo}-\mathrm{S}}\right)$ and S-Mo-Te $\left(A_{1}^{\mathrm{Mo}-\mathrm{Te}}\right)$ bond stretching, respectively [see the bottom-hand panel of Fig. 2(a)]. Moreover, the other two doubly degenerate optical-phonon modes are calculated to have frequencies 326.1 and $156.7 \mathrm{~cm}^{-1}$ and their vibrational characteristics are presented in the bottom-hand panel of Fig. 2(a). The $E^{\prime \text { Mo-S }}$ is attributed to the in-plane vibration of Mo and S atoms against each other while the $E^{\prime \prime M o-T e}$ reveals the inplane opposite vibration of the Te atom and the Mo-S couple. Notably, all of the four Raman-active modes possess different vibrational characteristics than those obtained in $1 \mathrm{H}$-MoX $(X=\mathrm{S}$ or $\mathrm{Te})$ structures. In contrast to individual $1 H-\mathrm{Mo} X_{2}$ monolayers, the center of mass of the individual atoms is not on the Mo layer in this Janus crystal. Total mass of Mo and $\mathrm{S}$ atoms is approximately equal to the mass of the Te atom and therefore the $A_{1}^{\mathrm{Mo}-\mathrm{Te}}$ and $E^{\prime \prime \mathrm{Mo}-\mathrm{Te}}$ phonon modes display likely the opposite vibration of two equal masses. However, when the Te atom does not contribute to the vibration, i.e., in $A_{1}^{\mathrm{Mo}-\mathrm{S}}$ and $E^{\text {Mo-S }}$ modes, then the center of the vibration is in between the Mo-S bonds.

The calculated Raman spectrum of Janus monolayer $1 T^{\prime}$ MoSTe is presented in Fig. 2(b) and the vibrational motion
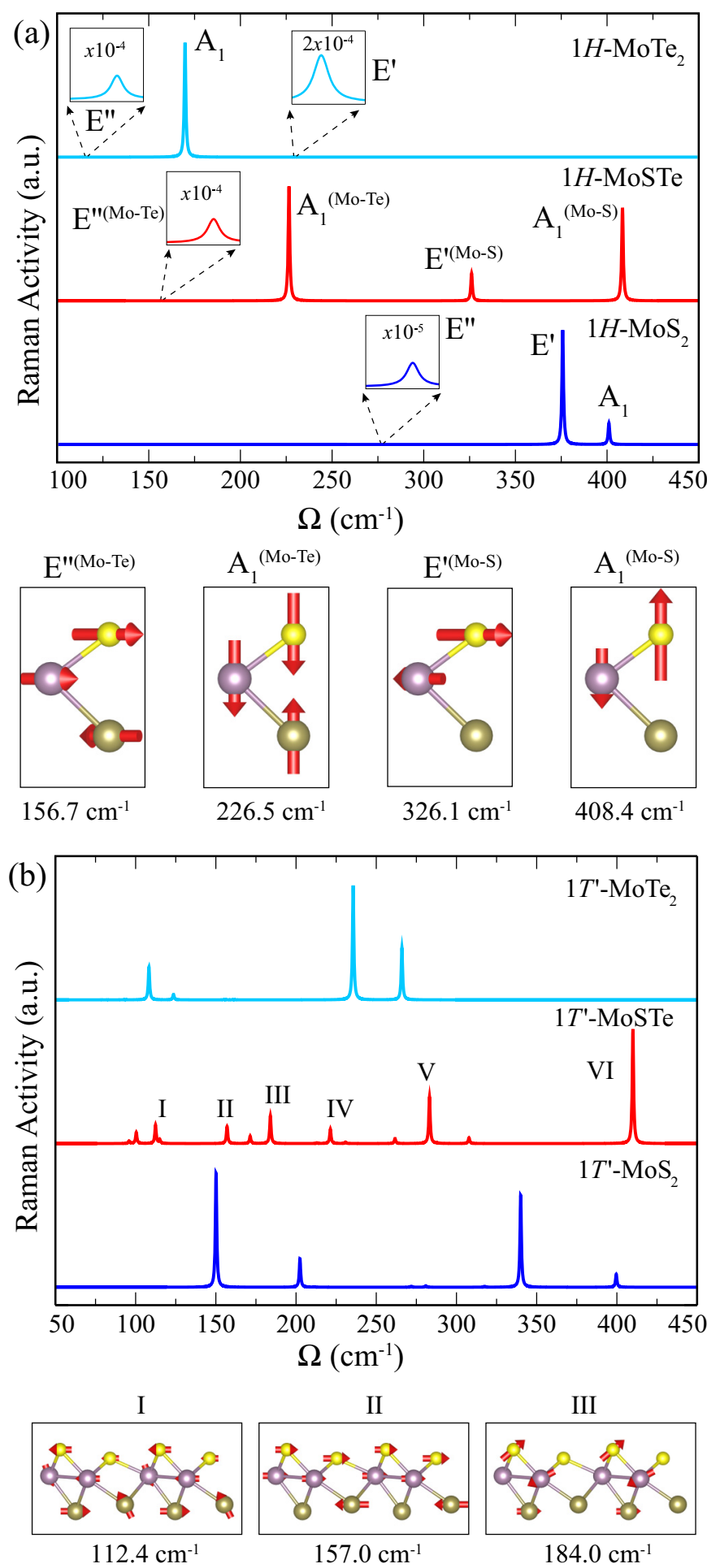

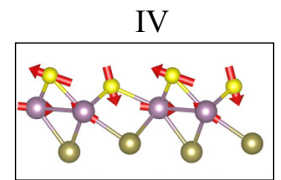

$221.4 \mathrm{~cm}^{-1}$

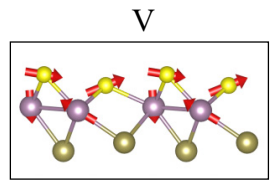

$283.2 \mathrm{~cm}^{-1}$

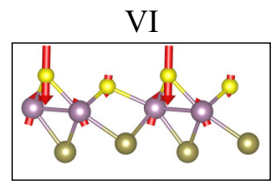

$410.0 \mathrm{~cm}^{-1}$
FIG. 2. For the monolayers of $\mathrm{MoS}_{2}, \mathrm{MoTe}_{2}$, and Janus MoSTe crystals, (a) Raman spectra for the $1 H$ phases and the vibrational characteristics of the Raman-active phonon modes. (b) Those for their $1 T^{\prime}$ phases. 
TABLE I. For the monolayer crystals $1 H$ - and $1 T^{\prime}$-MoSTe; the optimized lattice constants, $a$ and $b$, atomic bond lengths in the crystal, $d_{\mathrm{Mo-S}}$ and $d_{\mathrm{Mo}-\mathrm{Te}}$, the amount charge depletion, $\Delta_{\rho(\mathrm{Mo}-\mathrm{S})}$ and $\Delta_{\rho(\mathrm{Mo-Te})}$, energy band gaps calculated within GGA, $E_{\mathrm{gap}}^{\mathrm{GGA}}$, SOC on top of GGA, $E_{\text {gap }}^{\text {SOC }}$, and HSE on top of GGA+SOC, $E_{\text {gap }}^{\mathrm{HSE}}$, location of VBM and CBM edges in the BZ, the work functions calculated for two different surfaces, $\Phi_{\mathrm{S}}$ and $\Phi_{\mathrm{Te}}$, in-plane and out-of-plane dielectric constants, $\epsilon_{x x}, \epsilon_{y y}$, and $\epsilon_{z z}$, and frequencies of Raman-active phonon modes.

\begin{tabular}{|c|c|c|c|c|c|c|c|c|c|c|c|c|c|c|c|}
\hline Structural phase & $\begin{array}{c}a \\
(\AA)\end{array}$ & $\begin{array}{c}b \\
(\AA)\end{array}$ & $\begin{array}{c}d_{\mathrm{Mo-S}} \\
(\AA)\end{array}$ & $\begin{array}{c}d_{\mathrm{Mo}-\mathrm{Te}} \\
(\AA)\end{array}$ & $\begin{array}{c}\Delta_{\rho(\mathrm{Mo}-\mathrm{S})} \\
(e)\end{array}$ & $\begin{array}{c}\Delta_{\rho(\mathrm{Mo}-\mathrm{Te})} \\
\quad(e)\end{array}$ & $\begin{array}{l}E_{\mathrm{gap}}^{\mathrm{GGA}} \\
(\mathrm{eV})\end{array}$ & $\begin{array}{l}E_{\text {gap }}^{\mathrm{SOC}} \\
(\mathrm{eV})\end{array}$ & $\begin{array}{l}E_{\text {gap }}^{\mathrm{HSE}} \\
(\mathrm{eV})\end{array}$ & $\begin{array}{c}\Phi_{\mathrm{S}} \\
(\mathrm{eV})\end{array}$ & $\begin{array}{l}\Phi_{\mathrm{Te}} \\
(\mathrm{eV})\end{array}$ & VBM/CBM & $\begin{array}{c}\epsilon_{x x} \\
-\end{array}$ & $\begin{array}{c}\epsilon_{y y} \\
-\end{array}$ & $\begin{array}{c}\epsilon_{z z} \\
-\end{array}$ \\
\hline $1 H$-MoSTe & 3.36 & 3.36 & 2.43 & 2.72 & 0.6 & 0.2 & 1.06 & 1.04 & 1.50 & 5.12 & 4.46 & $\Gamma / \mathrm{K}$ & 4.84 & 4.84 & 1.23 \\
\hline
\end{tabular}

in some prominent Raman-active modes are shown in the bottom-hand panel. As compared to its $1 H$ phase, $1 T^{\prime}-\mathrm{MoSTe}$ exhibits more Raman-active modes (a total of 15 modes) some of which have low intensity. Similarly, additional Ramanactive modes appear in this structure due to the broken out-ofplane symmetry, when compared with the $1 T^{\prime}-M X_{2}$ crystals having four prominent peaks in their Raman spectra. As shown in the bottom-hand panel of Fig. 2(b), the Te atoms do not contribute to the vibrations which have higher frequencies. The most prominent peak having frequency 410.0 $\mathrm{cm}^{-1}$ (labeled as I) is dominated by the opposite out-of-plane vibration of the Mo and upper $S$ atoms while the lower $\mathrm{S}$ atoms have a smaller contribution to the vibration. The other two Raman-active modes having frequencies 283.2 and $221.4 \mathrm{~cm}^{-1}$, Te atoms do not have any contribution to the vibrations and those phonon modes reveal coupled in-plane and out-of-plane vibrations of Mo and S atoms. In another coupled phonon mode labeled as III, the upper Te, lower $\mathrm{S}$, and the some of the Mo atoms do not contribute to the vibration. The vibrational mode having frequency $157.0 \mathrm{~cm}^{-1}$ is completely attributed to the in-plane vibration of Te atoms against Mo-S couples. Note that here the lower Te atoms do not have contribution to the vibration. Last, the phonon mode labeled as I occurs at frequency $112.4 \mathrm{~cm}^{-1}$ and it is attributed to the in-plane opposite vibration of outer Te and $\mathrm{S}$ atoms with small contribution of Mo atoms. In addition, the inner Te atoms and their neighboring Mo atoms vibrate against each other in coupled in-plane and out-of-plane directions. It can be concluded that two different structural phases of Janus monolayer MoSTe structure exhibit totally different Raman spectra which are important to distinguish between them. Moreover, because the structural phase transitions between $1 H$ and $1 T^{\prime}$ phases of monolayer $\mathrm{MoTe}_{2}$ have been reported to be experimentally feasible, our results on the Raman spectra of Janus MoSTe phases are quite important to understand and observe such phase transition in even Janus MoSTe structure.

The Bader charge analysis shows that the two stable Janus MoSTe structures have similar charge characteristic such that Mo atom donates its $0.6 e^{-}$to a $\mathrm{S}$ atom and $0.2 e^{-}$to a Te atom (see Table I). The thermionic work function $(\Phi)$ is calculated from two different surfaces and the $\mathrm{S}$ side is found to have 5.12 and $5.28 \mathrm{eV}$, in $1 H$ - and $1 T^{\prime}-\mathrm{MoSTe}$, respectively. The work function from the Te side is calculated to be 4.46 and $5.76 \mathrm{eV}$, respectively, which displays the opposite trend in the two crystal phases.

\section{B. Electronic properties}

The electronic band structure and partial density of states (PDOS) of two dynamically stable Janus MoSTe crystals are investigated through the whole BZ. As seen in Figs. 2(a) and 2(b), the electronic properties of these two structures are apparently different due to the bonding states of Mo-S and Mo-Te atoms. Similar to the $1 H$ phases of monolayer $\mathrm{MoS}_{2}$ and $\mathrm{MoTe}_{2}$, Janus $1 H$-MoSTe exhibits semiconducting behavior with a band gap of $1.50 \mathrm{eV}$ but it is not a direct-gap semiconductor like $\mathrm{MoS}_{2}$ and $\mathrm{MoS}_{2}$, it is an indirect-gap semiconductor whose valence-band maximum (VBM) and conduction-band minimum (CBM) edges reside at the $\Gamma$ and $K$ points of the BZ, respectively.

A similar electronic transition was also predicted for the Janus monolayer of $\mathrm{In}_{2} \mathrm{SSe}$ crystal [23]. It was shown that the individual monolayers of InS and InSe are both indirect bandgap semiconductors while the constructed Janus monolayer $\mathrm{In}_{2} \mathrm{SSe}$ is a direct band-gap semiconductor. The source of such a different electronic structure was explained through the induced biaxial strain on the surfaces of the Janus crystal as mentioned above. In the case of the monolayer Janus $1 H$ MoSTe, the sulfur side experiences a tensile biaxial strain of $5.7 \%$ while the tellurium side is biaxially compressed by $4.8 \%$. However, with the $5.7 \%$ of biaxial tensile strain, monolayer $1 \mathrm{H}-\mathrm{MoS}_{2}$ turns into an indirect band-gap semiconductor for which the VBM shifts to the $\Gamma$ point. Similarly, the VBM of $1 \mathrm{H}-\mathrm{MoTe}_{2}$ displays a shift to the $\mathrm{M}$ point of the $\mathrm{BZ}$ via applied $4.8 \%$ of compressive biaxial strain. In both monolayers, the CBM edges remain at the $K$ point which is not significantly affected by the in-plane biaxial strain due to their out-of-plane orbital character. As seen in Fig. 3(a), the VBM of monolayer Janus $1 H$-MoSTe is dominated by the sulfur side of the Janus crystal. Moreover, the indirect band-gap nature of the monolayer $1 \mathrm{H}$-MoSTe can also be investigated through the band alignment of the strained monolayers by adjusting their vacuum energies. It is evident that the indirect nature of the band gap of $1 \mathrm{H}-\mathrm{MoSTe}$ is directly driven by the induced biaxial strains on both sides.

Distinctively, monolayer Janus $1 T^{\prime}$-MoSTe displays smallgap semiconductor character. The states in the vicinity of VBM are composed of S- $p$, Te- $p$, and are dominated by Mo- $d$ orbitals in both MoSTe phases. The CBM states are significantly dominated by Mo- $d$ orbitals in $1 H$ MoSTe while the $\mathrm{S}-p$ and Te- $p$ orbitals have remarkable contribution in $1 T^{\prime}$-MoSTe, as presented in Figs. 3(a) and 3(b). 

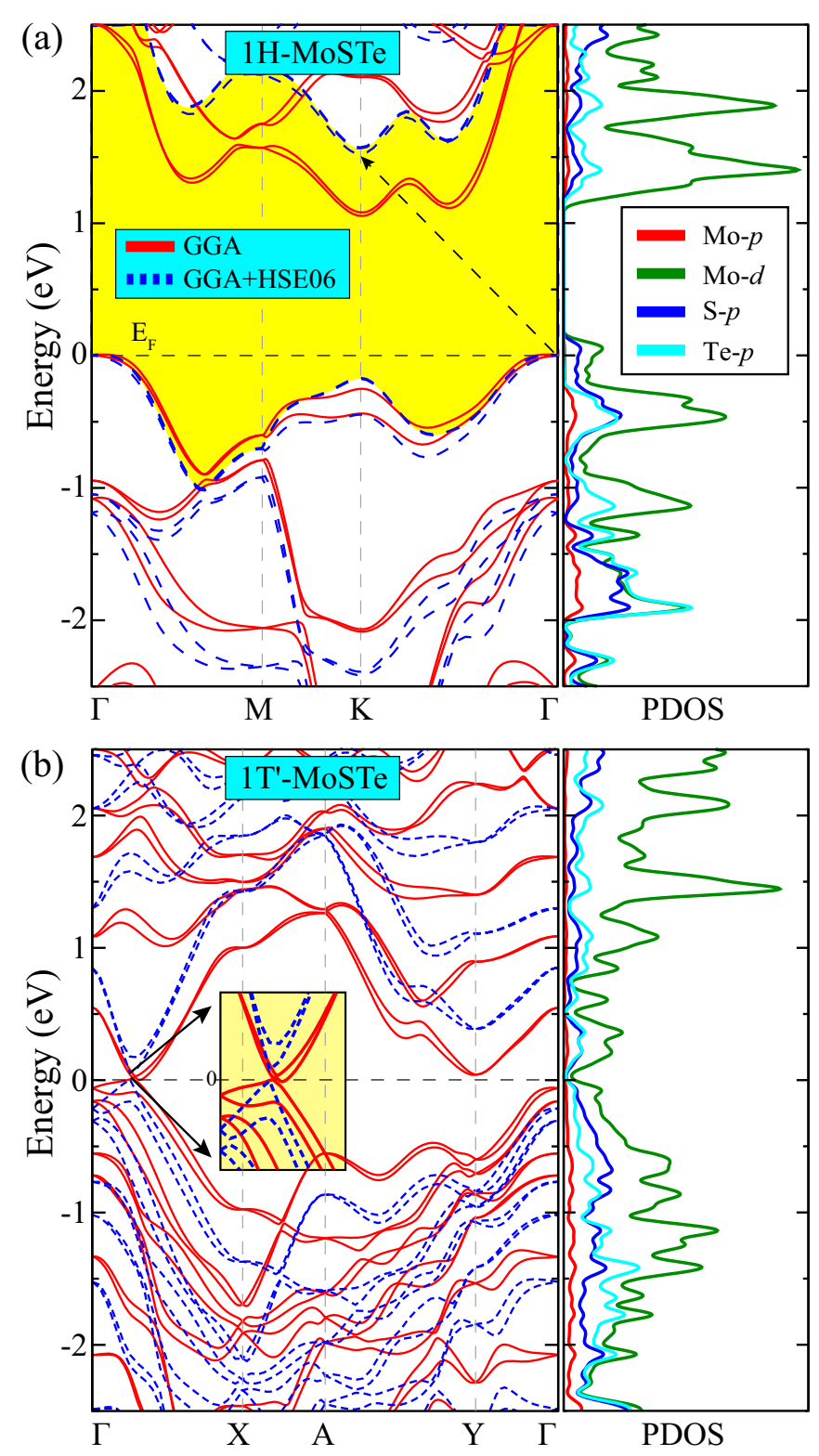

FIG. 3. The electronic band dispersions and the corresponding partial density of states (PDOS) of monolayer Janus; (a) $1 H$-MoSTe and (b) $1 T^{\prime}$-MoSTe. The Fermi level is set to zero.

\section{Linear-elastic properties}

The elastic properties of these ultrathin 2D materials can be represented by two independent constants: the in-plane stiffness $C$ and the Poisson ratio $v$. In order to determine the linear-elastic constants, the elastic strain tensor elements $C_{i j}$ are calculated and the corresponding $C$ and $v$ values are obtained for all orientations of the lattices however, those for only two main directions are listed in Table II. Note that among the listed stiffness parameters, the relaxed-ion ones are smaller than those for the clamped-ion case due to the release of the stress from the crystal after the ionic relaxations.

The in-plane stiffness is a measure of the rigidity of a material. Among 2D ultrathin materials, graphene is known to be the stiffest material with a large $C$ value $(330 \mathrm{~N} / \mathrm{m})$ [42]. Notably, the large stiffness value of graphene is directly related to the strong in-plane $s p^{2} \mathrm{C}-\mathrm{C}$ bonds. Due to the different bonding nature and type of atomic arrangement of the atoms, monolayer $\mathrm{MoS}_{2}$ is known to be slightly softer than graphene (with a stiffness of $122 \mathrm{~N} / \mathrm{m}$ ) [42]. Our calculations show that the monolayer Janus $1 H$-MoSTe possesses a stiffness of $109 \mathrm{~N} / \mathrm{m}$ which is in between those for $1 H-\mathrm{MoS}_{2}(122$ $\mathrm{N} / \mathrm{m})$ and $1 H-\mathrm{MoTe}_{2}(83 \mathrm{~N} / \mathrm{m})$. In comparison, $1 H-\mathrm{MoSTe}$ is a considerably stiff material except for graphene and $h-\mathrm{BN}$. On the other hand, $1 T^{\prime}-\mathrm{MoSTe}$ is slightly anisotropic due to the Mo-Mo chains and the calculated in-plane stiffness values parallel and perpendicular to the chains are 92 and $90 \mathrm{~N} / \mathrm{m}$, respectively. As listed in Table II, the construction of the Janus monolayer decreases the anisotropy between the two main orientations. The in-plane stiffness values of the two directions are more anisotropic for $1 T^{\prime}-\mathrm{MoS}_{2}$ (112 and $123 \mathrm{~N} / \mathrm{m})$ and for $1 T^{\prime}-\mathrm{MoTe}_{2}(44$ and $76 \mathrm{~N} / \mathrm{m})$. It is clear that the exchange of one surface of $1 T^{\prime}-\mathrm{MoTe}_{2}$ by $\mathrm{S}$ atoms makes the Mo-Mo bonds stronger and thus the in-plane stiffness is significantly enhanced.

In addition, the orientation-dependent elastic constants are also calculated by using the following equations [43];

$$
\begin{aligned}
C(\theta) & =\frac{\left(C_{11} C_{22}-C_{12}^{2}\right)}{C_{22} \cos ^{4}(\theta)+A \cos ^{2}(\theta) \sin ^{2}(\theta)+C_{11} \sin ^{4}(\theta)}, \\
\nu(\theta) & =\frac{C_{12} \cos ^{4}(\theta)-B \cos ^{2}(\theta) \sin ^{2}(\theta)+C_{12} \sin ^{4}(\theta)}{C_{22} \cos ^{4}(\theta)+A \cos ^{2}(\theta) \sin ^{2}(\theta)+C_{11} \sin ^{4}(\theta)},
\end{aligned}
$$

TABLE II. For the $1 H$ and $1 T^{\prime}$ phases of monolayers $\mathrm{MoS}_{2}, \mathrm{MoTe}_{2}$, and MoSTe; the relaxed- and clamped-ion elastic coefficients $C_{i j}$, the

\begin{tabular}{|c|c|c|c|c|c|c|c|c|c|c|c|c|c|c|}
\hline $\begin{array}{l}\text { Crystal } \\
\text { Structure }\end{array}$ & \multicolumn{5}{|c|}{ Relaxed ion } & \multicolumn{9}{|c|}{ Clamped ion } \\
\hline $1 H-\mathrm{MoS}_{2}$ & 131 & 131 & 34 & 122 & 122 & 0.26 & 0.26 & 155 & 155 & 51 & 138 & 138 & 0.33 & 0.33 \\
\hline $1 H-\mathrm{MoTe}_{2}$ & 88 & 88 & 21 & 83 & 83 & 0.24 & 0.24 & 111 & 111 & 35 & 100 & 100 & 0.32 & 0.32 \\
\hline $1 H-\mathrm{MoSTe}$ & 116 & 116 & 28 & 109 & 109 & 0.24 & 0.24 & 140 & 140 & 44 & 126 & 126 & 0.31 & 0.31 \\
\hline $1 T^{\prime}-\mathrm{MoSTe}$ & 98 & 101 & 29 & 90 & 92 & 0.30 & 0.29 & 128 & 132 & 46 & 112 & 116 & 0.36 & 0.35 \\
\hline
\end{tabular}
corresponding in-plane stiffness $C$, and Poisson ratio $v$. 


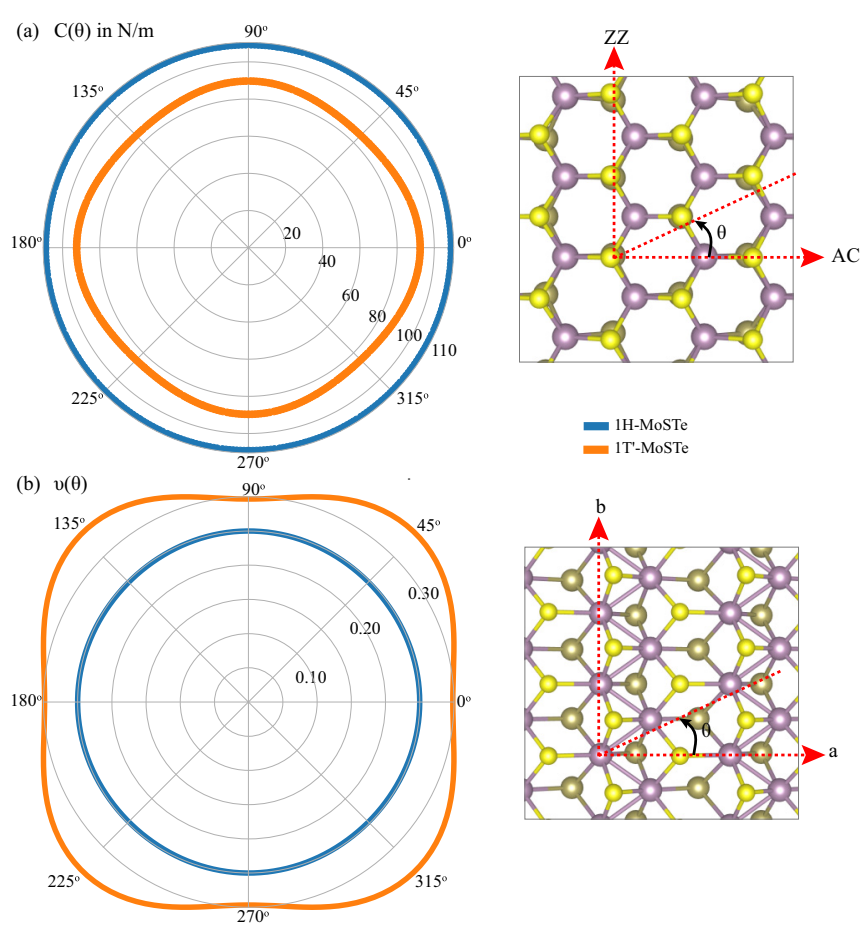

FIG. 4. For the monolayers of Janus $1 H$ - and $1 T^{\prime}$-MoSTe crystals the orientation angle-dependent (a) in-plane stiffness $C(\theta)$ and (b) Poisson ratio $v(\theta) . A C$ and $Z Z$ in $1 H$ phase stand for the armchair and zigzag directions, respectively, while $a$ and $b$ in $1 T^{\prime}$ phase represent the directions perpendicular and parallel to the Mo chains, respectively.

where the numbers $A$ and $B$ are defined as $A=\left(C_{11} C_{22}-\right.$ $\left.C_{12}^{2}\right) / C_{66}-2 C_{12}$ and $B=C_{11}+C_{22}-\left(C_{11} C_{22}-C_{12}^{2}\right) / C_{66}$. Notably, the in-plane stiffness along arbitrary orientations can be obtained using the above equations. Specifically, the $1 T^{\prime}$ phase of Janus MoSTe is expected to possess such orientation dependency of the elastic constants due to its anisotropic nature.

The mechanical response of a material to external stress is known as the Poisson ratio which is defined as the ratio of the transverse contraction strain to the longitudinal extension. The Poisson ratio $v$ is calculated to be 0.24 for monolayer Janus $1 H$-MoSTe which is approximately the same as that of $1 H-\mathrm{MoTe}_{2}$ and slightly smaller than that of $1 H-\mathrm{MoS}_{2}(0.26)$. In addition, the $1 T^{\prime}$-MoSTe displays very small anisotropy as compared to the MoSTe [see Fig. 4(b)]. The Poisson ratio values are calculated to be 0.29 and 0.30 along parallel and perpendicular directions to the Mo-Mo chains. These values are significantly larger than those for monolayer $1 T^{\prime}-\mathrm{MoS}_{2}$ $(0.15$ and 0.14 , respectively) and smaller than those for $1 T^{\prime}-\mathrm{MoTe}_{2}$ (0.55 and 0.39 , respectively). The values indicate the strong ability of monolayer Janus $1 T^{\prime}$-MoSTe to preserve its equilibrium state under external applied strain.

\section{Piezoelectric properties}

Piezoelectric effect is known as the generating electric dipole moment as a result of applied mechanical stress in noncentrosymmetric materials. Theoretical predictions and experimental observations have demonstrated the enhancement of piezoelectric constants in two dimensions [44-47].

In the 2D limit, the relaxed-ion piezoelectric tensor $e_{i j}$ can be described as the sum of ionic $e_{i j}^{\text {ion }}$ and electronic $e_{i j}^{\mathrm{el}}$ contributions. The piezoelectric stress tensor $e_{i j}$ is related to the piezoelectric strain tensor $d_{i j}$ through the elastic stiffness tensor $C_{i j}$ as follows:

$$
e_{i j}=d_{i k} C_{k j} \text {. }
$$

As already reported before, for the $1 H$-phase structures the piezoelectric strain coefficients $d_{i j}$ are given by the relations

$$
d_{11}=\frac{e_{11}}{C_{11}-C_{12}}, \quad d_{13}=\frac{e_{13}}{C_{11}+C_{12}} .
$$

Notably, for $1 H-\mathrm{MoS}_{2}$ and $1 H-\mathrm{MoTe}_{2}$ the $d_{13}$ is found to be zero because of the inversion symmetry along the outof-plane direction. However, in the case of the Janus $1 \mathrm{H}$ MoSTe structure, breaking the out-of-plane symmetry adds an additional degree of freedom and the $d_{13}$ is no longer zero. As listed in Table III, the $d_{11}$ coefficient for the $1 H$ MoSTe is $4.5 \times 10^{-10} \mathrm{C} / \mathrm{m}$ which is in between those for $\mathrm{MoS}_{2}\left(3.7 \times 10^{-10} \mathrm{C} / \mathrm{m}\right)$ and $\mathrm{MoTe}_{2}\left(4.7 \times 10^{-10} \mathrm{C} / \mathrm{m}\right)$. The existence of different chalcogen atoms at each side breaks the mirror symmetry in the out-of-plane direction. Therefore, the $e_{13}$ is calculated to be $0.5 \times 10^{-10} \mathrm{C} / \mathrm{m}$ for $1 H$-MoSTe. The corresponding piezoelectric strain coefficients are calculated by using Eq. (4) and the $d_{11}$ and $d_{13}$ are found to be 5.1 and $0.4 \mathrm{pm} / \mathrm{V}$, respectively. In the case of Janus $1 T^{\prime}$ $\mathrm{MoST}$, the central symmetry existing in $\mathrm{MoS}_{2}$ and $\mathrm{MoTe}_{2}$ (therefore they are not piezoelectric in any direction) is broken due to different chalcogen sides. Therefore, four nonzero components are found for the piezoelectric stress tensor which are the $e_{14}, e_{21}$, and $e_{22}$. As listed in Table III, their values are calculated to be $-5.7,4.0$, and $-8.3\left(\times 10^{-10} \mathrm{C} / \mathrm{m}\right)$ which are considerably large values as compared to $e_{11}$ of the $1 H$ phase. The corresponding piezoelectric strain values are calculated using the equations

$$
\begin{gathered}
d_{14}=\frac{e_{14}}{C_{44}}, \\
d_{21}=\frac{e_{21} C_{22}-e_{22} C_{12}}{C_{11} C_{22}-C_{12}^{2}}, \\
d_{22}=\frac{e_{22} C_{11}-e_{21} C_{21}}{C_{11} C_{22}-C_{12}^{2}} .
\end{gathered}
$$

The piezoelectric strain coefficients $d_{14}, d_{21}$, and $d_{22}$ are found to be $-17.8,7.1$, and $-10.3 \mathrm{pm} / \mathrm{V}$, respectively.

\section{CONCLUSION}

In this study, the electronic, vibrational, and piezoelectric properties of dynamically stable two phases of monolayer Janus MoSTe, namely $1 H$-MoSTe and $1 T^{\prime}$-MoSTe, were investigated. Our results on phonon band dispersions revealed that apart from its $1 T$ phase, monolayer Janus MoSTe possesses dynamical stability in its $1 H$ and $1 T^{\prime}$ phases. As the exchange of a lighter chalcogen atom via a heavier one is energetically favorable, the formation of $1 T^{\prime}$-MoSTe seems to be feasible if monolayer $1 T^{\prime}-\mathrm{MoTe}_{2}$ is used as the basis structure. Raman spectrum calculations indicated that the two phases of Janus MoSTe exhibit totally different vibrational features which allows one to distinguish the two phases. 
TABLE III. For the $1 H$ and $1 T^{\prime}$ phases of monolayers $\mathrm{MoS}_{2}, \mathrm{MoTe}_{2}$, and MoSTe, the relaxed-ion piezoelectric stress $e_{i j}$ and the corresponding piezoelectric strain $d_{i j}$ coefficients.

\begin{tabular}{|c|c|c|c|c|c|c|c|c|c|c|}
\hline $\begin{array}{l}\text { Crystal } \\
\text { Structure }\end{array}$ & $\begin{array}{c}e_{11} \\
\left(10^{-10} \mathrm{C} / \mathrm{m}\right)\end{array}$ & $\begin{array}{c}e_{13} \\
\left(10^{-10} \mathrm{C} / \mathrm{m}\right)\end{array}$ & $\begin{array}{c}e_{14} \\
\left(10^{-10} \mathrm{C} / \mathrm{m}\right)\end{array}$ & $\begin{array}{c}e_{21} \\
\left(10^{-10} \mathrm{C} / \mathrm{m}\right)\end{array}$ & $\begin{array}{c}e_{22} \\
\left(10^{-10} \mathrm{C} / \mathrm{m}\right)\end{array}$ & $\begin{array}{c}d_{11} \\
(\mathrm{pm} / \mathrm{V})\end{array}$ & $\begin{array}{c}d_{13} \\
(\mathrm{pm} / \mathrm{V})\end{array}$ & $\begin{array}{c}d_{14} \\
(\mathrm{pm} / \mathrm{V})\end{array}$ & $\begin{array}{c}d_{21} \\
(\mathrm{pm} / \mathrm{V})\end{array}$ & $\begin{array}{c}d_{22} \\
(\mathrm{pm} / \mathrm{V})\end{array}$ \\
\hline $1 H-\mathrm{MoS}_{2}$ & 3.7 & & & & & 3.8 & & & & \\
\hline $1 H$-MoSTe & 4.5 & 0.5 & & & & 5.1 & 0.4 & & & \\
\hline \multicolumn{11}{|l|}{$1 T^{\prime}-\mathrm{MoS}_{2}$} \\
\hline \multicolumn{11}{|l|}{$1 T^{\prime}-\mathrm{MoTe}_{2}$} \\
\hline
\end{tabular}

Moreover, electronic band dispersions revealed that the $1 H$ MoSTe is found to be an indirect band-gap semiconductor while $1 T^{\prime}$-MoSTe is small-gap semiconductor. Notably, the indirect band-gap nature of the Janus monolayer was shown to arise due to the induced surface strains on each side of the structure. In addition, calculated elastic tensors revealed the slightly anisotropic nature of the distorted phase in terms of the in-plane stiffness. We further analyzed the piezoelectric properties of the phases and it is found that while the S-Te exchange creates an out-of-plane piezoelectric coefficient in $1 H$-MoSTe, the $1 T^{\prime}$ phase turns into a piezoelectric material due to broken out-of-plane symmetry. Overall, our find- ings indicated the energetic feasibility, dynamical stability, anisotropic elastic nature, and the created piezoelectric properties of $1 T^{\prime}$-MoSTe.

\section{ACKNOWLEDGMENTS}

Computational resources were provided by TUBITAK ULAKBIM, High Performance and Grid Computing Center (TR-Grid e-Infrastructure). This work was supported by the Flemish Science Foundation (FWO-Vl) by a postdoctoral fellowship (M.Y.).
[1] S. K. Novoselov, A. K. Geim, S. V. Morozov, D. Jiang, Y. Zhang, S. V. Dubonos, I. Grigorieva, and A. A. Firsov, Science 306, 666 (2004).

[2] A. H. C. Neto and K. Novoselov, Rep. Prog. Phys. 74, 082501 (2011).

[3] K. F. Mak, C. Lee, J. Hone, J. Shan, and T. F. Heinz, Phys. Rev. Lett. 105, 136805 (2010).

[4] A. Splendiani, L. Sun, Y. Zhang, T. Li, J. Kim, C.-Y. Chim, G. Galli, and F. Wang, Nano Lett. 10, 1271 (2010).

[5] B. Radisavljevic, A. Radenovic, J. Brivio, i. V. Giacometti, and A. Kis, Nat. Nanotechnol. 6, 147 (2011)

[6] J. T. Ye, Y. J. Zhang, R. Akashi, M. S. Bahramy, R. Arita, and Y. Iwasa, Science 338, 1193 (2012).

[7] R. V. Kasowski, Phys. Rev. Lett. 30, 1175 (1973).

[8] L. F. Mattheis, Phys. Rev. Lett. 30, 784 (1973).

[9] L. F. Mattheis, Phys. Rev. B 8, 3719 (1973).

[10] S. Lebegue and O. Eriksson, Phys. Rev. B 79, 115409 (2009).

[11] H. S. S. Ramakrishna Matte, A. Gomathi, A. K. Manna, D. J. Late, R. Datta, S. K. Pati, and C. N. R. Rao, Angew. Chem. 122, 4153 (2010).

[12] K. Liu, Q. Yan, M. Chen, W. Fan, Y. Sun, J. Suh, D. Fu, S. Lee, J. Zhou, S. Tongay, J. Ji, J. B. Neaton, and J. Wu, Nano Lett. 14, 5097 (2014).

[13] J. A. Wilson and A. D. Yoffe, Adv. Phys. 18, 193 (1969).

[14] X. Qian, J. Liu, L. Fu, and J. Li, Science 346, 1344 (2014).

[15] Y. Ma, L. Kou, X. Li, Y. Dai, S. C. Smith, and T. Heine, Phys. Rev. B 92, 085427 (2015).

[16] D. Voiry, A. Mohite, and M. Chhowalla, Chem. Soc. Rev. 44, 2702 (2015).

[17] D. Rhodes, D. A. Chenet, B. E. Janicek, C. Nyby, Y. Lin, W. Jin, D. Edelberg, E. Mannebach, N. Finne, A. Antony, T. Schiros, T.
Klarr, A. Mazzoni, M. Chin, Y.-c Chiu, W. Zheng, Q. R. Zhang, F. Ernst, J. I. Dadap, X. Tong, J. Ma, R. Lou, S. Wang, T. Qian, H. Ding, R. M. Osgood, D. W. Paley, A. M. Lindenberg, P. Y. Huang, A. N. Pasupathy, M. Dubey, J. Hone, and L. Balicas, Nano Lett. 17, 1616 (2017).

[18] S. Cho, S. Kim, J. Ho Kim, J. Zhao, J. Seok, D. H. Keum, J. Baik, D.-H. Choe, K. J. Chang, K. Suenaga, S. W. Kim, Y. H. Lee, and H. Yang, Science 349, 625 (2015).

[19] D. H. Keum, S. Cho, J. H. Kim, D. H. Choe, H. J. Sung, M. Kan, H. Kang, J. Y. Hwang, S. W. Kim, H. Yang, K. J. Chang, and Y. H. Lee, Nat. Phys. 11, 482 (2015).

[20] Y. Wang, J. Xiao, H. Zhu, Y. Li, Y. Alsaid, K. Y. Fong, Y. Zhou, S. Wang, W. Shi, Y. Wang, A. Zettl, E. J. Reed, and X. Zhang, Nature (London) 550, 487 (2017).

[21] A.-Y. Lu, H. Zhu, J. Xiao, C.-P. Chuu, Y. Han, M.-H. Chiu, C.-C. Cheng, C.-W. Yang, K.-H. Wei, Y. Yang, Y. Wang, D. Sokaras, D. Nordlund, P. Yang, D. A. Muller, M-Y. Chou, X. Zhang, and L.-J. Li, Nat. Nanotechnol. 12, 744 (2017).

[22] J. Zhang, S. Jia, I. Kholmanov, L. Dong, D. Er, W. Chen, H. Guo, Z. Jin, V. B. Shenoy, L. Shi, and J. Lou, ACS Nano 11, 8192 (2017).

[23] A. Kandemir and H. Sahin, Phys. Rev. B 97, 155410 (2018).

[24] Y. C. Cheng, Z. Y. Zhu, M. Tahir, and U. Schwingenschlögl, Europhys. Lett. 102, 57001 (2013).

[25] L. Dong, J. Lou, and V. B. Shenoy, ACS Nano 11, 8242 (2017).

[26] H. Jin, T. Wang, Z.-R. Gong, C. Long, and Y. Dai, Nanoscale 10, 19310 (2018).

[27] W. Shi and Z. Wang, J. Phys.: Condens. Matter. 30, 215301 (2018).

[28] C. Xia, W. Xiong, J. Du, T. Wang, Y. Peng, and J. Li, Phys. Rev. B 98, 165424 (2018). 
[29] H. Liu, Z. Huang, C. He, Y. Wu, L. Xue, C. Tang, X. Qi, and J. Zhong, Appl. Phys. Lett. 125, 082516 (2019).

[30] S.-D. Guo and J. Dong, Semicond. Sci. Technol. 33, 085003 (2018).

[31] Y. Sun, Z. Shuai, and D. Wang, Nanoscale 10, 21629 (2018).

[32] J. P. Perdew, K. Burke, and M. Ernzerhof, Phys. Rev. Lett. 77, 3865 (1996).

[33] J. P. Perdew, K. Burke, and M. Ernzerhof, Phys. Rev. Lett. 78, 1396 (1997).

[34] G. Kresse and J. Hafner, Phys. Rev. B 47, 558 (1993).

[35] G. Kresse and J. Hafner, Phys. Rev. B 49, 14251 (1994).

[36] J. Heyd, G. E. Scuseria, and M. Ernzerhof, J. Chem. Phys. 118, 8207 (2003).

[37] S. J. Grimme, Comput. Chem. 27, 1787 (2006).

[38] G. Henkelman, A. Arnaldsson, and H. Jonsson, Comput. Mater. Sci. 36, 354 (2006).
[39] D. Alfe, Comput. Phys. Commun. 180, 2622 (2009).

[40] M. Yagmurcukardes, C. Bacaksiz, E. Unsal, B. Akbali, R. T. Senger, and H. Sahin, Phys. Rev. B 97, 115427 (2018).

[41] M. Yagmurcukardes, F. M. Peeters, and H. Sahin, Phys. Rev. B 98, 085431 (2018).

[42] M. Yagmurcukardes, R. T. Senger, F. M. Peeters, and H. Sahin, Phys. Rev. B 94, 245407 (2016).

[43] Y. Ma, L. Kou, B. Huang, Y. Dai, and T. Heine, Phys. Rev. B 98, 085420 (2018).

[44] M. N. Blonsky, H. L. Zhuang, A. K. Singh, and R. G. Hennig, ACS Nano 9, 9885 (2015).

[45] M. M. Alyoruk, Y. Aierken, D. Cakir, F. M. Peeters, and C. Sevik, J. Phys. Chem. C 119, 23231 (2015).

[46] R. Fei, W. Li, and J. Li, Appl. Phys. Lett. 107, 173104 (2015).

[47] L. C. Gomes, A. Carvalho, and A. H. Castro Neto, Phys. Rev. B 92, 214103 (2015). 